Results Four studies were included, out of 2693 records. One hundred and thirty-seven pregnancies were recorded in 102 women with mean age ranging from 27.2 years to 39.9 years in 1992-2018. At conception, the predominant organ manifestations were renal and cutaneous, whereas disease activity measures were generally unavailable. Livebirths occurred in $57 \%-82 \%$ of pregnancies and pregnancy loss in $18 \%-43 \%$. Other major adverse pregnancy outcomes (figure 1) were: SLE flare mainly presented as nephritis, pre-eclampsia (9\%-33\%), pre-term birth (up to $56 \%$ of livebirths), and low birthweight (14\%-85\% of live births). No study clearly reported risk factors for adverse pregnancy outcomes. Rather, the factors likely associated with adverse obstetric, fetal and neonatal outcomes included SLE flare (especially nephritis), maternal serological status during pregnancy and hypertensive disorders of pregnancy.

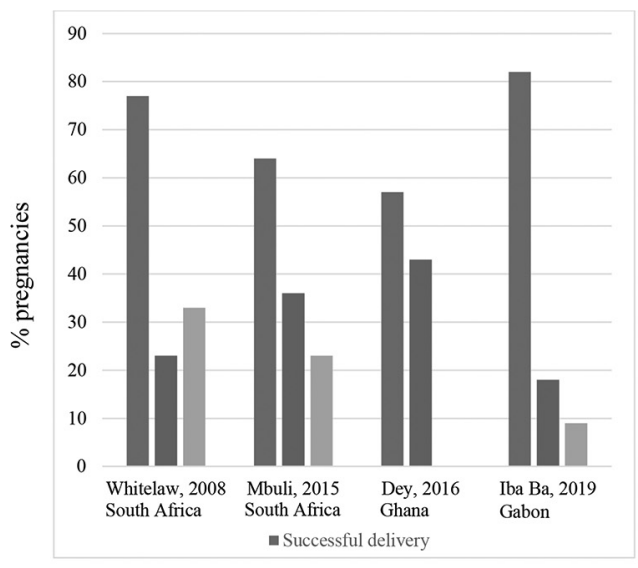

A. Obstetric complications

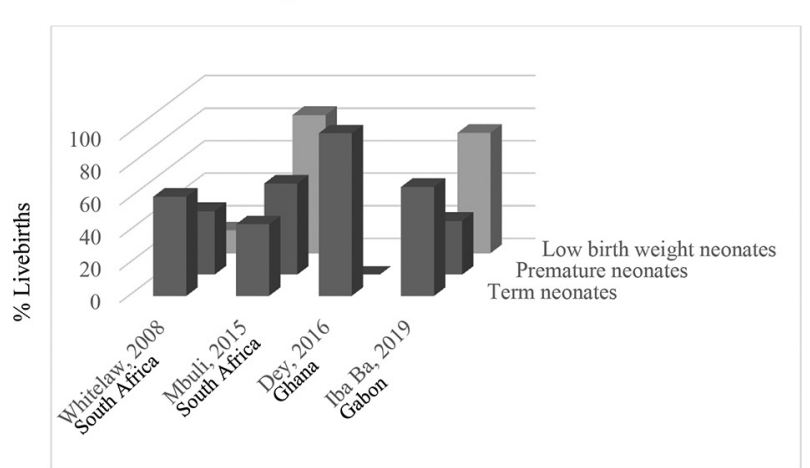

B. Neonatal complications

Abstract P78 Figure 1 Main complications of systemic lupus erythematosus pregnancies in sub-Saharan Africa in 1992-2018.

Conclusions Contemporary available literature reports overall low rates of livebirth in sub-Saharan African women with SLE. The main adverse pregnancy outcomes reported are : SLE flare, pregnancy loss, pre-eclampsia, pre-term birth and low birthweight. Although no risk factor has been clearly mentioned, SLE flare presented as nephritis is the major factor likely associated with adverse pregnancy outcomes. Thorough investigation is warranted.

Acknowledgements None.

\section{P79 PREDICTORS OF ADVERSE NEONATAL OUTCOME DURING THE PREGNANCY OF WOMEN WITH ANTIPHOSPHOLIPID SYNDROME IN THE FRENCH GR2 PROSPECTIVE STUDY}

${ }^{1}$ Anne Murarasu, ${ }^{1}$ Gaëlle Guettrot-Imbert, ${ }^{1}$ Véronique Le Guern, ${ }^{2}$ Francois Maurier, ${ }^{3}$ Patrick Jego, ${ }^{4}$ Estibaliz Lazaro, ${ }^{5}$ Alban Deroux, ${ }^{6}$ Maëlle Le Besnerais, ${ }^{7}$ Odile SouchaudDebouverie, ${ }^{8}$ Pauline Orquevaux, ${ }^{9}$ Catherine Deneux-Tharaux, ${ }^{1}$ Nathalie CostedoatChalumeau, the GR2 study group. 'Internal Medicine Dept., Hôpital Cochin AP-HP, Paris; ${ }^{2}$ Internal Medicine Dept., Hôpital Belle-Isle, Metz; 'Internal Medicine Dept., CHU de Rennes, Rennes; ${ }^{4}$ Internal Medicine Dept., CHU de Bordeaux, Bordeaux; ${ }^{5}$ Internal Medicine Dept., CHU de Grenoble, La Tronche; ${ }^{6}$ Internal Medicine Dept., CHU de Rouen, Rouen; ${ }^{7}$ Internal Medicine Dept., CHU de la Milétrie, Poitiers; ${ }^{8}$ Internal Medicine Dept., CHU Robert Debré, Reims; ${ }^{9}$ EPOPé Team, University of Paris, Epidemiology and Statistics Research Center/ CRESS, INSERM, INRA F-75004, Paris, France

\subsection{6/lupus-2020-eurolupus.124}

Introduction Data about the predictors of adverse neonatal outcome in pregnant women with antiphospholipid syndrome (APS) are sparse. The main study on this subject is the prospective American study PROMISSE on 144 carriers of antiphospholipid antibodies. ${ }^{1}$ We report the first results of the French study, GR2.

Methods Inclusion criteria were: (1) an ongoing pregnancy at 12 weeks of gestation (WG), (2) conceived before May 2018, (3) of a woman with APS. ${ }^{2}$ Exclusion criteria were proteinuria (ratio $>1 \mathrm{~g} / \mathrm{g}$ ), serum creatinine $>100 \mu \mathrm{mol} / \mathrm{L}$, and multifetal pregnancy.

The composite primary outcome included an intrauterine fetal death (IUFD), a neonatal death, placental insufficiency (fetal growth restriction, preeclampsia, HELLP syndrome, and/ or placental abruption) leading to a delivery $\leq 34 \mathrm{WG}$, and/or a small for gestational age (SGA) $\leq 3$ rd percentile.

Results We analysed 119 pregnancies in 119 patients: 53\% thrombotic and 47\% obstetric only APS; 60\% had lupus anticoagulant (LA) and 26\% associated SLE. Treatment included aspirin (99\%), heparin (98\%, in the therapeutic range for $50 \%)$, and hydroxychloroquine $(62 \%)$.

The primary outcome was observed in $14 \%$ of pregnancies: $3 \%$ IUFD, $8 \%$ delivery $\leq 34 \mathrm{WG}$ due to placental insufficiency and/or 5\% of SGA (and no neonatal death). It was observed in $20 \%$ of the pregnancies with LA compared with $7 \%$ for women negative for LA $(P=0.096)$. This outcome did not differ according to anticardiolipin or antiß2GP1 antibody status, or prior thrombosis (14\%, versus $14 \%$ for women with only obstetric APS). There was a trend toward more adverse neonatal outcomes in case of prior arterial thrombosis $(29 \%$ versus $12 \%, P=0.07)$. The rate of adverse neonatal outcomes did not differ among the women with or without associated SLE (29\% versus $26 \%, P=0.77$ ).

In multivariate analysis, independent features associated with pregnancy failure were: in a serological model, LA positive status $(\mathrm{ORa}=8.5,95 \% \mathrm{CI}[1.0-71.5])$ and in a serological and clinical model, 'non-white' skin colour $(\mathrm{ORa}=6.1,95 \% \mathrm{CI}$ [1.1-34.7]).

Conclusion The rate of adverse neonatal outcomes tended to be lower than in the PROMISSE study (14\% versus 19\%), although the women included here were theoretically more severe (definite APS). One potential explanation might be that our patients were all treated. In keeping with the PROMISSE study, LA and skin colour were the main predictors of neonatal outcome.

Acknowledgement Fondation pour la Recherche Médicale (M2R201806006403) 